\title{
Multiparticle potentials from lightlike Wilson lines in quark-gluon plasmas: A generalized relation of in-medium splitting rates to jet-quenching parameters $\hat{q}$
}

\author{
Peter Arnold \\ Department of Physics, University of Virginia, Charlottesville, Virginia 22904-4714, USA
}

(Received 24 January 2019; published 22 March 2019)

\begin{abstract}
A powerful historical insight about the theory of in-medium showering in QCD backgrounds was that splitting rates can be related to a parameter $\hat{q}$ that characterizes the rate of transverse-momentum kicks to a high-energy particle from the medium. Another powerful insight was that $\hat{q}$ can be defined (with caveats) even when the medium is strongly coupled, using long, narrow Wilson loops whose two long edges are lightlike Wilson lines. The medium effects for the original calculations of in-medium splitting rates can be formulated in terms of three-body imaginary-valued "potentials" that are defined with three long, lightlike Wilson lines. Corrections due to the overlap of two consecutive splittings can be calculated using similarly defined four-body potentials. I give a simple argument for how such $N$-body potentials can be determined in the appropriate limit just from the knowledge of the values of $\hat{q}$ for different color representations. For $N>3$, the $N$-body potentials have a nontrivial color structure, which will complicate calculations of overlap corrections outside of the large- $N_{\mathrm{c}}$ or soft bremsstrahlung limits.
\end{abstract}

DOI: 10.1103/PhysRevD.99.054017

\section{OVERVIEW AND RESULT}

In theoretical studies of $p_{\perp}$ broadening and jet quenching of very high-energy partons that travel through a quarkgluon plasma, a very important parameter describing scattering of the parton from the medium is known as $\hat{q}$. Physically, it is the proportionality constant in the relation $\left\langle Q_{\perp}^{2}\right\rangle=\hat{q} \Delta z$, where $\left\langle Q_{\perp}^{2}\right\rangle$ is the typical squared transverse momentum (transverse to the parton's initial direction of motion) that the parton picks up after traveling a distance $\Delta z$ through the medium, in the limit that $\Delta z$ is large compared to characteristic scales of the medium such as mean-free paths for collisions. As I briefly review later, it has been known for some time [1,2] that $\hat{q}$ can also be formally defined (with important caveats) in terms of a kind of "potential energy" $V(\Delta b)$ defined using a mediumaveraged Wilson loop having two long parallel lightlike sides separated by transverse distance $\Delta b$, as shown in Fig. 1(a). This definition is similar to how potentials are often defined for static charges using Wilson loops such as Fig. 1(b). The potential $V(\Delta b)$ is extracted from the exponential dependence of the Wilson loop on its length,

Published by the American Physical Society under the terms of the Creative Commons Attribution 4.0 International license. Further distribution of this work must maintain attribution to the author(s) and the published article's title, journal citation, and DOI. Funded by SCOAP ${ }^{3}$.

$$
\left\langle\operatorname{tr}\left[P e^{i g \oint_{C} d x^{\mu} A_{\mu}}\right]\right\rangle \approx e^{-i V(\Delta b) L}
$$

where $P$ represents path ordering in color space. ${ }^{1}$ Formally (again with important caveats), one can show that $\hat{q}$ is the coefficient of a harmonic oscillator approximation to this potential,

$$
V(\Delta b) \rightarrow-\frac{i}{4} \hat{q}(\Delta b)^{2} \text { for small } \Delta b,
$$

which is equivalent to

$$
\left\langle\operatorname{tr}\left[P e^{i g} \oint_{C} d x^{\mu} A_{\mu}\right]\right\rangle \approx e^{-\hat{q}(\Delta b)^{2} L / 4} \quad \text { for small } \Delta b .
$$

The advantage of the Wilson loop language is that it can be used as a tool for discussing $\hat{q}$ in strongly (as well as weakly) coupled quark-gluon plasmas. In general, $\hat{q}$ depends on the color representation $R$ of the high-energy particle. For weakly coupled plasmas, $\hat{q}$ is simply proportional to the quadratic Casimir $C_{R}$ of that color representation, but for strongly coupled plasmas the $\hat{q}$ for different color representations may not be so simply related. ${ }^{2}$

\footnotetext{
${ }^{1}$ My $L$ corresponds to the $L^{-} / \sqrt{2}$ of Refs. [1,2], and my transverse separation $\Delta b$ is what they call $L$. My characterization of the Wilson loop as defining a "potential" $V(\Delta \boldsymbol{b})$ is not language specifically used by Refs. [1,2].

${ }^{2}$ The Casimir scaling $\hat{q}_{R} \propto C_{R}$ holds through next-to-leading order in the strength of the coupling of the plasma [3], but this scaling need not hold exactly at all orders. For some examples of violation of Casimir scaling for Wilson loops in other contexts, see Refs. [4-6].
} 


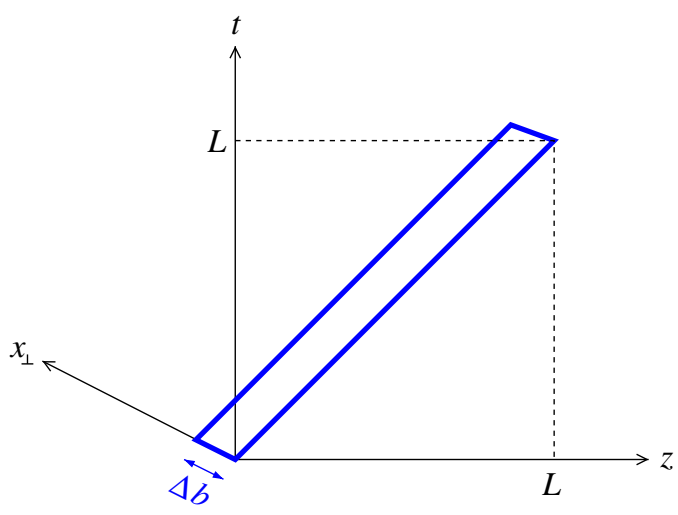

(a)

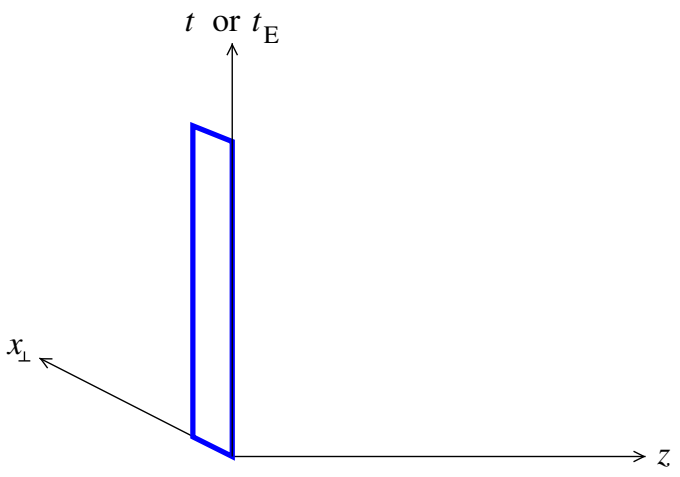

(b)

FIG. 1. (a) A Wilson loop with lightlike edges used to formally define $\hat{q}$ (subject to caveats mentioned in Sec. IV). $t$ is real (Minkowski) time. In contrast, (b) shows a Wilson loop for static color charges, where $t$ can be real or imaginary (Euclidean) time.

Similar types of potentials arise in calculations of splitting rates (bremsstrahlung or pair production) in high-energy in-medium showers. Splitting rate calculations are complicated by the Landau-Pomeranchuk-Migdal (LPM) effect, which accounts for the fact that high-energy particles can scatter from the medium many times during the quantum mechanical duration, known as the formation time, of a single splitting. The QCD version of the LPM effect was originally worked out by Baier et al. (BDMPS) [7,8] and Zakharov [9]. Though they originally framed their calculations in terms of a weakly coupled picture of the medium, the approach can be generalized to a strongly coupled medium. Consider the left-hand side of Fig. 2, which depicts an interference term that contributes to an inmedium splitting rate. Following Zakharov [9], one may sew together the diagrams representing the amplitude and conjugate amplitude to form the interference diagram on the right-hand side, which may now be formally reinterpreted as the propagation of three particles through the medium, where particles from the conjugate diagram (red lines) are reinterpreted as their antiparticles. Zakharov

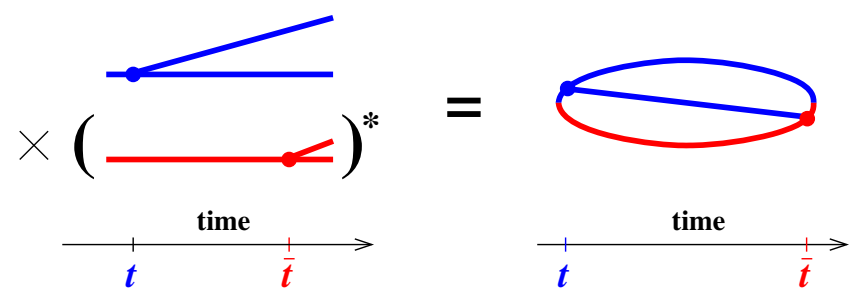

FIG. 2. A contribution to the rate for single splitting of a highenergy particle in the medium. Only high-energy particles are shown explicitly; all lines are implicitly interacting with the medium, which is then averaged over. In these diagrams, time runs from left to right, and the amplitude and conjugate amplitude are each implicitly integrated over the time of emission $(t$ and $\bar{t}$, respectively) to get the splitting probability. On the right-hand side is a combined diagram showing the amplitude (blue lines) sewn together with the conjugate amplitude (red lines). expresses the calculation of this interference in terms of the time evolution of the wave function of the transverse positions of the three particles, which can be described by a Schrödinger-like equation. The potential term in that Schrödinger equation represents medium-averaged effects of interactions with the medium over timescales short compared to the long formation time. Over those timescales, the transverse positions of the particles can be treated as constant. We could therefore identify this potential term as the three-particle potential $V\left(\boldsymbol{b}_{1}, \boldsymbol{b}_{2}, \boldsymbol{b}_{3}\right)$ between lightlike Wilson lines, such as depicted in Fig. 3. In the high-energy limit, the relevant separations $\boldsymbol{b}_{i}-\boldsymbol{b}_{j}$ are small during the formation time because splitting processes

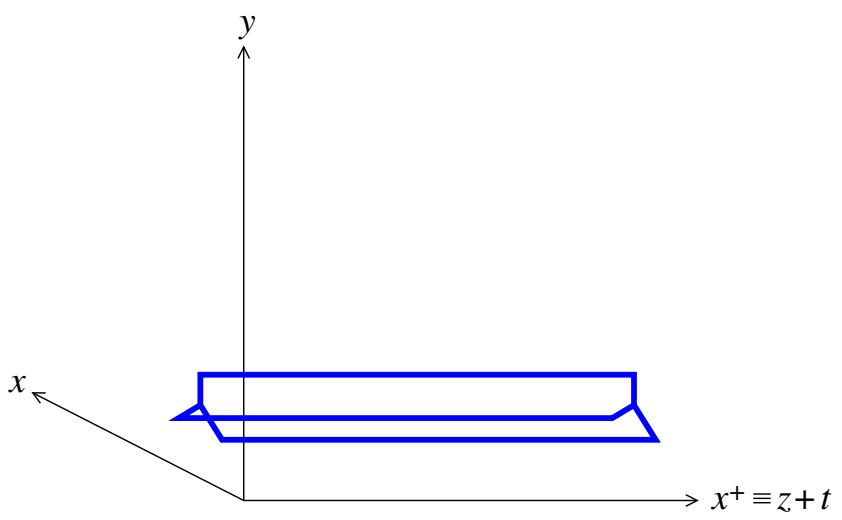

FIG. 3. As Fig. 1(a) but for a three-body potential. Note that the axes are depicted differently than in Fig. 1: in order to be able to show both transverse spatial directions, the $t$ and $z$ axes have been collapsed to $x^{+}=z+t$, with $x^{-}=0$ everywhere. The threepoint vertices on the ends are chosen to form the color-neutral combination of the three particles. For example, for the threegluon potential, the Wilson lines would be adjoint representation and the three-point vertices would each be proportional to the Lie algebra structure constants $f^{a b c}$. Note: The constant transverse positions $\left(\boldsymbol{b}_{1}, \boldsymbol{b}_{2}, \boldsymbol{b}_{3}\right)$ of the three lightlike Wilson lines can be anything; they need not be symmetrically arranged as in this picture. 
are nearly collinear, and so one may make a harmonic oscillator approximation to $V\left(\boldsymbol{b}_{1}, \boldsymbol{b}_{2}, \boldsymbol{b}_{3}\right)$. As I will review, there is a fairly simple argument $[10]^{3}$ that, whenever a harmonic oscillator approximation is relevant, the approximation must necessarily have the form

$$
\begin{aligned}
V\left(\boldsymbol{b}_{1}, \boldsymbol{b}_{2}, \boldsymbol{b}_{3}\right)= & -\frac{i}{8}\left[\left(\hat{q}_{1}+\hat{q}_{2}-\hat{q}_{3}\right)\left(\boldsymbol{b}_{2}-\boldsymbol{b}_{1}\right)^{2}\right. \\
& +\left(\hat{q}_{2}+\hat{q}_{3}-\hat{q}_{1}\right)\left(\boldsymbol{b}_{3}-\boldsymbol{b}_{2}\right)^{2} \\
& \left.+\left(\hat{q}_{3}+\hat{q}_{1}-\hat{q}_{2}\right)\left(\boldsymbol{b}_{1}-\boldsymbol{b}_{3}\right)^{2}\right],
\end{aligned}
$$

where $\hat{q}_{1}, \hat{q}_{2}$, and $\hat{q}_{3}$ are the $\hat{q}$ 's for the color representations of the three high-energy particles involved in the splitting process (the parent and the two daughters). The argument for (1.4) is simple in the sense that it does not require any of the machinery of computing the LPM effect in QCD: it just involves thinking through the constraints to any harmonic three-body potential from the special cases where some of the separations $\boldsymbol{b}_{i}-\boldsymbol{b}_{j}$ vanish.

There has been a variety of work on the LPM effect in QCD studying the potentially significant effects of what happens when two consecutive splittings in an in-medium shower have overlapping formation times. As I will briefly review, such calculations generally require corresponding four-particle potentials $V\left(\boldsymbol{b}_{1}, \boldsymbol{b}_{2}, \boldsymbol{b}_{3}, \boldsymbol{b}_{4}\right)$. Including yet more particles would be needed to study the simultaneous overlap of three or more splittings. To date, calculations of overlap effects have made simplifying assumptions such as soft emission limits [11-13] or the large- $N_{\mathrm{c}}$ limit $[10,14-16]$. In this paper, I take a first step toward removing those assumptions by finding the generalization of (1.4) to four or more particles. The result will be

$$
\underline{V}\left(\boldsymbol{b}_{1}, \boldsymbol{b}_{2}, \ldots, \boldsymbol{b}_{N}\right)=-\frac{i}{8} \sum_{i>j}\left(\hat{q}_{i}+\hat{q}_{j}-\underline{\hat{q}}_{i j}\right)\left(\boldsymbol{b}_{i}-\boldsymbol{b}_{j}\right)^{2},
$$

where underlines indicate an operator on the space of color states of the $N$ particles. The color structure is necessary because of the $\hat{q}_{i j}$ above, which refers to the combined color representation of particles $i$ and $j$. If both are gluons, for example, the combined color representation could be any irreducible representation $R$ in the $\mathrm{SU}(3)$ tensor product $8 \otimes 8=1 \oplus 8 \oplus 8 \oplus 10 \oplus \overline{10} \oplus 27$, and those representations generally have different values of $\hat{q}$. The color operator $\underline{\hat{q}}_{i j}$ represents the use of the correct value of $\hat{q}$ in each color subspace. Formally,

\footnotetext{
${ }^{3}$ See in particular the discussion in Appendix A of Ref. [10] concerning Eq. (2.21) of that paper. In actual splitting rate calculations, one can use symmetries (like BDMPS and Zakharov did) to reduce the work of solving the three-body problem to solving an effective one-body problem with a potential derived from (1.4). For a description in the language used here, see Secs. II.E and III of Ref. [10].
}
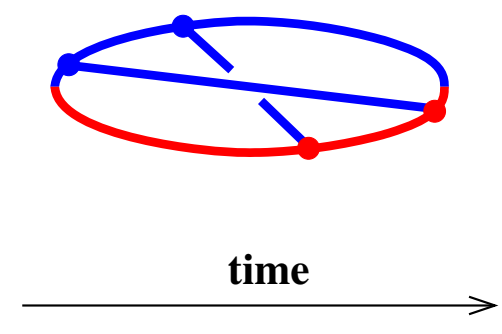

FIG. 4. Similar to the right-hand side of Fig. 2 but for double splitting.

$$
\underline{\hat{q}}_{i j}=\sum_{R \in R_{i} \otimes R_{j}} \hat{q}_{R} \underline{\mathcal{P}}_{i j, R}
$$

where $\underline{\mathcal{P}}_{i j, R}$ is a projection operator, acting on the $N$-particle color space, that selects the subspace where particles $i$ and $j$ have combined (irreducible) color representation $R$.

Since the particles described by the potential (1.5) are separated from each other (unless some $\boldsymbol{b}_{i}=\boldsymbol{b}_{j}$ ), readers may be concerned about whether, in the general case, the "combined color representation" of any pair of particles $i$ and $j$ is a gauge-invariant concept. I will later discuss the separation of scales in this problem that addresses this point.

If one chooses a basis of the $N$-particle color space where each basis element can be identified as belonging to a particular irreducible combined color representation of particles 1 and 2, then $\hat{q}_{12}$ can be represented as a diagonal matrix on the space of colors. However, in that basis, $\hat{q}_{13}$ will generally not be diagonal. As a result, the potential $\underline{V}$ of (1.5) generically contains terms which mix different possible color combinations of the $N$ particles.

The reason that this complication concerning color representations was avoided in the three-particle potential (1.4) has to do with the overall color state of the particles in the applications of interest. Consider Zakharov's interpretation of the single splitting process, depicted by the righthand side of Fig. 2. The total color of the $N=3$ particles there is neutral. The same is automatically true of any gauge-invariant definition of a three-body potential from Wilson lines, such as in Fig. 3. For $N=3$, overall color neutrality means that the combined color representation of particles 1 and 2 must be the conjugate of the color representation of particle 3 , so that $\hat{q}_{12}=\hat{q}_{3}$ and similarly for permutations. This is how the $N$-body result (1.5) reduces to the simpler three-body result (1.4) for $N=3$.

Figure 4 similarly shows an example of an interference term for the process of double splitting, in a case relevant to computing effects of overlapping formation times [10]. For part of the time evolution in this example, there are $N=4$ particles, also forming an overall color singlet. For $N>3$, this overall color neutrality does not constrain the combined color representations of each pair of particles to a unique irreducible representation, and so the matrix structure of the potential $\underline{V}$ in color space is unavoidable. 
In general, $N$-body potentials for overall color singlets are a tool for consolidating all interactions with the medium that occur over timescales small compared to the timescale of the splitting processes shown in Figs. 2 and 4, i.e., on timescales small compared to formation times.

\section{A. Outline}

Before proceeding to a general argument for the $N$-body potential (1.5), it may help motivate the color structure of the result to first discuss the special case of weakly coupled plasmas in Sec. II. There I start with a brief review of the relation of $\hat{q}$ to the two-body potential, and then generalize to a discussion of the $N$-body potential. That section is not necessary, though, for readers wishing to quickly cut to the chase and see the general argument for the result (1.5), which is given in Sec. III.

In Sec. IV, I list many of the caveats that I have been sweeping under the rug concerning the definition and meaning of $\hat{q}$ and harmonic oscillator approximations to the potential. I also explain the hierarchy of timescales that makes it sensible to discuss a potential with nontrivial color structure. Section V offers a brief recap and conclusion.

\section{THE SPECIAL CASE OF WEAKLY COUPLED PLASMAS}

\section{A. Two-body potential and $\hat{\boldsymbol{q}}$}

First, I start with a brief review of the physics behind the two-body potential. It is useful to forget about Wilson loops for a moment and first review the probabilistic evolution of the $\boldsymbol{p}_{\perp}$ of a high-energy particle receiving random transverse momentum kicks as it crosses the plasma. ${ }^{4}$

\section{Evolution of transverse momentum}

For a high-energy particle, we can follow the evolution of the classical probability distribution $\rho\left(\boldsymbol{p}_{\perp}\right)$ of its transverse momentum using the equation

$$
\frac{\partial \rho\left(\boldsymbol{p}_{\perp}, t\right)}{\partial t}=-\int d^{2} q_{\perp} \frac{d \Gamma_{\mathrm{el}}}{d^{2} q_{\perp}}\left[\rho\left(\boldsymbol{p}_{\perp}, t\right)-\rho\left(\boldsymbol{p}_{\perp}-\boldsymbol{q}_{\perp}, t\right)\right]
$$

where $d \Gamma_{\mathrm{el}} / d^{2} q_{\perp}$ is the differential rate for the high-energy particle to pick up transverse momentum $\boldsymbol{q}_{\perp}$ from the medium. The first term on the right-hand side of (2.1) is a loss term, corresponding to the chance for momentum $\boldsymbol{p}_{\perp}$ to be scattered to some other momentum; the second term is a gain term, corresponding to some other momentum

\footnotetext{
${ }^{4}$ The review in Sec. II A 1 is a generalization of BDMPS's Eqs. (2.8)-(2.12) and (3.1) of Ref. [8]. By casting the derivation in terms of $d \Gamma_{\mathrm{el}} / d^{2} q_{\perp}$ [17] instead of BDMPS's $V\left(Q^{2}\right)$, the review here avoids BDMPS's model assumption that the medium can be treated as a collection of static scattering centers.
}

scattering to become $\boldsymbol{p}_{\perp}$. The equation can be solved by Fourier transforming from $\boldsymbol{p}_{\perp}$ to transverse position space,

$$
\frac{\partial \rho(\boldsymbol{b}, t)}{\partial t}=-\Delta \Gamma_{\mathrm{el}}(\boldsymbol{b}) \rho(\boldsymbol{b})
$$

where

$\Delta \Gamma_{\mathrm{el}}(\boldsymbol{b}) \equiv \Gamma_{\mathrm{el}}(0)-\Gamma_{\mathrm{el}}(\boldsymbol{b})=\int d^{2} q_{\perp} \frac{d \Gamma_{\mathrm{el}}}{d^{2} q_{\perp}}\left(1-e^{i \boldsymbol{b} \cdot \boldsymbol{q}_{\perp}}\right)$

and

$$
\Gamma_{\mathrm{el}}(\boldsymbol{b}) \equiv \int d^{2} q_{\perp} \frac{d \Gamma_{\mathrm{el}}}{d^{2} q_{\perp}} e^{i \boldsymbol{b} \cdot \boldsymbol{q}_{\perp}}
$$

Multiplying both sides of (2.2) by $i$, the evolution equation may be formally recast as a Schrödinger-like equation

$$
i \partial_{t} \rho(\boldsymbol{b}, t)=V(\boldsymbol{b}) \rho(\boldsymbol{b}, t)
$$

with no kinetic term and with imaginary-valued potential energy

$$
V(\boldsymbol{b})=-i \Delta \Gamma_{\mathrm{el}}(\boldsymbol{b})
$$

The corresponding solution is

$$
\rho(\boldsymbol{b}, t)=e^{-i V(\boldsymbol{b}) t} \rho(\boldsymbol{b}, 0)=e^{-\Delta \Gamma_{\mathrm{el}}(\boldsymbol{b}) t} \rho(\boldsymbol{b}, 0) .
$$

\section{The same potential from a Wilson loop}

At leading order in the weak-coupling limit (with resummation of in-medium self-energies), this same physics arises from lightlike Wilson loops via two-point correlators of interactions of the Wilson lines with background gauge fields, as depicted in Fig. 5. Those correlators between Wilson lines can be shown to correspond to $\Gamma_{\mathrm{el}}\left(\boldsymbol{b}_{i}-\boldsymbol{b}_{j}\right)$. (For the sake of completeness, I review this in Appendix A, but the details will not be important.) Each self-energy loop on a Wilson line is additionally associated with a factor ${ }^{5}$ of $-\frac{1}{2}$, and so the exponent in Fig. 5 is $-i V(\Delta \boldsymbol{b}) L$ with

\footnotetext{
${ }^{5}$ When integrating over the relative time $\Delta x^{+}$between the two end points of a two-point correlator in Fig. 5, the integral is $\int_{-\infty}^{\infty} d\left(\Delta x^{+}\right)$for correlators that span two different Wilson lines $(i \neq j)$ but, to avoid double counting of a given loop, is instead $\int_{0}^{\infty} d\left(\Delta x^{+}\right)=\frac{1}{2} \int_{-\infty}^{\infty} d\left(\Delta x^{+}\right)$for self-energy corrections to a single Wilson line $(i=j)$. The relative minus sign for selfenergies compared to correlations between different Wilson lines is because the color path ordering of the two Wilson lines is in opposite directions. (Equivalently, if one wants to view both lines as running forward in time, then it is because the color charges $\mathbb{T}_{1}$ and $\mathbb{T}_{2}$ of the two lines must be opposite by overall color neutrality $\mathbb{T}_{1}+\mathbb{T}_{2}=0$ of the Wilson loop. See Sec. II B.)
} 


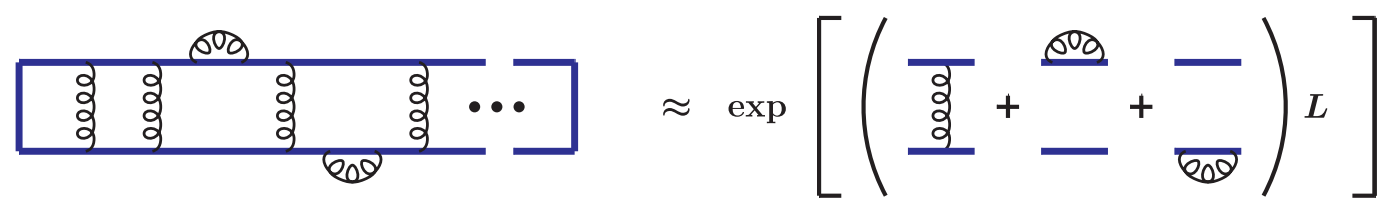

FIG. 5. The Wilson loop in the weak-coupling limit. Here, the gluon lines represent two-point correlators of the gauge field in the background of the medium. The particular graph on the left-hand side is just an example. The important point is that, in the weakcoupling limit, localized nonoverlapping two-point correlations dominate and exponentiate as shown on the right-hand side. Each twopoint correlation (depicted by a gluon line) is implicitly resummed with in-medium self-energy insertions.

$$
V(\Delta \boldsymbol{b})=-i\left[\Gamma_{\mathrm{el}}(0)-\Gamma_{\mathrm{el}}(\Delta \boldsymbol{b})\right],
$$

reproducing the potential (2.6).

In this weak-coupling limit, the only dependence on the color representation of the high-energy particle is that $d \Gamma_{\mathrm{el}}$ is proportional to $g^{2} C_{R}$, where $C_{R}$ is the quadratic Casimir of that representation. It will be useful in the remaining discussion of the weakly coupled limit to explicitly pull out this Casimir and write

$\Gamma_{\mathrm{el}}=C_{R} \bar{\Gamma}_{\mathrm{el}}, \quad V(\Delta \boldsymbol{b})=C_{R} \bar{V}(\Delta \boldsymbol{b}), \quad \hat{q}=C_{R} \hat{\bar{q}}, \quad$ etc.

where the barred quantities do not (in weak coupling) depend on the color representation of the high-energy particle.

\section{Relation to $\hat{q}$}

Formally expanding (2.3) in powers of $\boldsymbol{b}$, the small- $b$ limit is

$$
V(\boldsymbol{b}) \simeq-\frac{i}{4} b^{2} \int d^{2} q_{\perp} \frac{d \Gamma_{\mathrm{el}}}{d^{2} q_{\perp}} q_{\perp}^{2} .
$$

This is related to $\hat{q}$ : The rate at which the $p_{\perp}^{2}=\mid \boldsymbol{q}_{\perp 1}+$ $\boldsymbol{q}_{\perp 2}+\left.\cdots\right|^{2}$ of a hard particle would increase with time from an initial $p_{\perp}^{2} \equiv 0$, by a sequence of random kicks $\boldsymbol{q}_{\perp}$ from the medium, is given by

$$
\hat{q}=\int d^{2} q_{\perp} \frac{d \Gamma_{\mathrm{el}}}{d^{2} q_{\perp}} q_{\perp}^{2}
$$

So $(2.10)$ is

$$
V(\boldsymbol{b}) \simeq-\frac{i}{4} \hat{q} b^{2} .
$$

\section{B. The $N$-body potential}

Figure 6 shows a similar set of correlators for an $N$-body potential. The charge with which Wilson line $i$ interacts with a background gauge field of adjoint color $a$ is $g \underline{\mathbb{I}}_{i}^{a}$, where the $\mathbb{\mathbb { I }}_{i}^{a}$ are color generators in the color representation of particle $i$, acting on the color space of that particular particle. In the weak-coupling limit, Fig. 6 shows that the $N$-body potential then decomposes into two-body correlators as

$$
\begin{aligned}
& V\left(\boldsymbol{b}_{1}, \boldsymbol{b}_{2}, \ldots, \boldsymbol{b}_{N}\right) \\
& \quad=-i\left\{\frac{1}{2} \sum_{i} \underline{\mathbb{I}}_{i}^{2} \bar{\Gamma}_{\mathrm{el}}(0)+\sum_{i>j} \underline{\mathbb{I}}_{i} \cdot \underline{\mathbb{T}}_{j} \bar{\Gamma}_{\mathrm{el}}\left(\boldsymbol{b}_{i}-\boldsymbol{b}_{j}\right)\right\},
\end{aligned}
$$

which is the generalization of (2.8). Above, $\underline{\mathbb{T}}_{i} \cdot \underline{\mathbb{T}}_{j}$ represents the sum $\underline{\mathbb{Z}}_{i}^{a} \mathbb{\mathbb { T }}_{j}^{a}$ over $a$. Since for the applications of interest the collection of $N$ particles is overall color neutral, we can subtract $0=-\frac{i}{2}\left(\underline{\mathbb{I}}_{1}+\underline{\mathbb{Z}}_{2}+\cdots+\underline{\mathbb{I}}_{N}\right)^{2} \bar{\Gamma}_{\text {el }}(0)$ from (2.13) to rewrite it in the form

$$
\begin{aligned}
V\left(\boldsymbol{b}_{1}, \boldsymbol{b}_{2}, \ldots, \boldsymbol{b}_{N}\right) & =i \sum_{i>j} \underline{\mathbb{T}}_{i} \cdot \underline{\mathbb{I}}_{j} \Delta \bar{\Gamma}_{\mathrm{el}}\left(\boldsymbol{b}_{i}-\boldsymbol{b}_{j}\right) \\
& =-\sum_{i>j} \underline{\mathbb{T}}_{i} \cdot \underline{\mathbb{I}}_{j} \bar{V}_{(2)}\left(\boldsymbol{b}_{i}-\boldsymbol{b}_{j}\right),
\end{aligned}
$$

where $\bar{V}_{(2)}$ is the universal (in weak coupling) two-body potential when the color generators are factored out. For small transverse separations, Eq. (2.12) then gives

$$
V\left(\boldsymbol{b}_{1}, \boldsymbol{b}_{2}, \ldots, \boldsymbol{b}_{N}\right) \simeq \frac{i}{4} \sum_{i>j} \underline{\mathbb{I}}_{i} \cdot \underline{\mathbb{I}}_{j} \hat{\bar{q}}\left(\boldsymbol{b}_{i}-\boldsymbol{b}_{j}\right)^{2} .
$$

Rewriting

$$
\begin{aligned}
\underline{\mathbb{I}}_{i} \cdot \underline{\mathbb{I}}_{j} & =\frac{1}{2}\left[\left(\underline{\mathbb{I}}_{i}+\underline{\mathbb{I}}_{j}\right)^{2}-\underline{\mathbb{I}}_{i}^{2}-\underline{\mathbb{I}}_{j}^{2}\right] \\
& =\frac{1}{2}\left[\left(\underline{\mathbb{I}}_{i}+\underline{\mathbb{I}}_{j}\right)^{2}-C_{i}^{2}-C_{j}^{2}\right],
\end{aligned}
$$

and remembering that $\hat{q}_{R}=C_{R} \hat{\bar{q}}=\mathbb{\mathbb { I }}_{R}^{2} \hat{\bar{q}}$ (in weak coupling), the potential (2.15) can be recast as

$$
\underline{V}\left(\boldsymbol{b}_{1}, \boldsymbol{b}_{2}, \ldots, \boldsymbol{b}_{N}\right)=-\frac{i}{8} \sum_{i>j}\left(\hat{q}_{i}+\hat{q}_{j}-\underline{\hat{q}}_{i j}\right)\left(\boldsymbol{b}_{i}-\boldsymbol{b}_{j}\right)^{2} .
$$

This demonstrates the result (1.5) in the special case of weak coupling. 


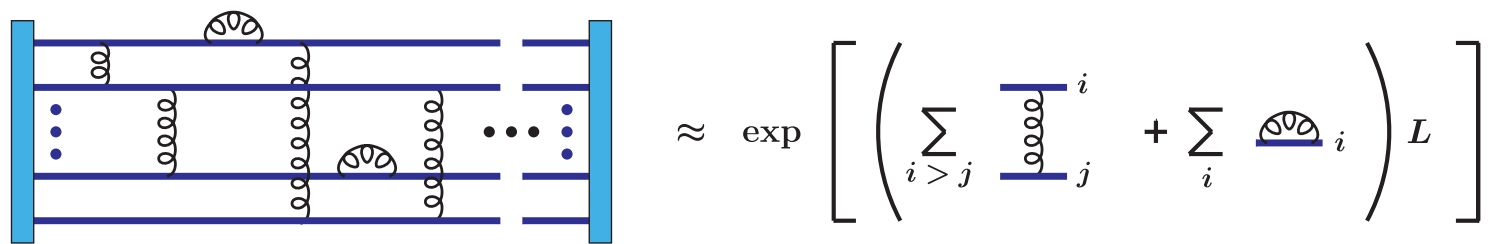

FIG. 6. As Fig. 5 but for an $N$-body potential. The blue rectangles indicate some contraction of the lightlike Wilson lines to form a gauge-invariant quantity (and so an overall color-neutral state of the $N$ high-energy particles represented by the Wilson lines).

\section{GENERAL ARGUMENT FOR ANY STRENGTH COUPLING}

Generically, if a function $V\left(\boldsymbol{b}_{1}, \ldots, \boldsymbol{b}_{N}\right)$ is translationally and rotationally invariant (here in the transverse plane) and approaches a smooth limit as all the $\boldsymbol{b}_{i}$ become coincident, we may suspect that it can be Taylor expanded in powers of $\boldsymbol{b}_{i}-\boldsymbol{b}_{j}$ to obtain a harmonic oscillator approximation in the limit of small separations. Here, we now give a simple argument why, if there is such a harmonic oscillator approximation, then its form is fixed as (1.5), provided the $N$ particles in total form a color singlet.

Start by assuming a generic harmonic oscillator form that is (transversely) translation invariant:

$$
\underline{V}\left(\boldsymbol{b}_{1}, \boldsymbol{b}_{2}, \ldots, \boldsymbol{b}_{N}\right)=\sum_{i j k l} \underline{c}_{i j k l}\left(\boldsymbol{b}_{i}-\boldsymbol{b}_{j}\right) \cdot\left(\boldsymbol{b}_{k}-\boldsymbol{b}_{l}\right)
$$

for some constants $\underline{c}_{i j k l}$. Those constants must be allowed to be color operators, since we have already seen that is necessary in the special case (2.15) of weak coupling. Using the algebraic identity

$$
\begin{aligned}
& \left(\boldsymbol{b}_{i}-\boldsymbol{b}_{j}\right) \cdot\left(\boldsymbol{b}_{k}-\boldsymbol{b}_{l}\right) \\
& =\frac{1}{2}\left[\left(\boldsymbol{b}_{i}-\boldsymbol{b}_{l}\right)^{2}+\left(\boldsymbol{b}_{j}-\boldsymbol{b}_{k}\right)^{2}-\left(\boldsymbol{b}_{i}-\boldsymbol{b}_{k}\right)^{2}-\left(\boldsymbol{b}_{j}-\boldsymbol{b}_{l}\right)^{2}\right],
\end{aligned}
$$

any potential of the form (3.1) can be recast into the form

$$
\underline{V}\left(\boldsymbol{b}_{1}, \boldsymbol{b}_{2}, \ldots, \boldsymbol{b}_{N}\right)=\sum_{i>j} \underline{a}_{i j}\left(\boldsymbol{b}_{i}-\boldsymbol{b}_{j}\right)^{2}
$$

for some constants $\underline{a}_{i j}$.

\section{A. The three-particle case}

Before continuing with the $N$-particle case, it will be helpful to review the logic of the three-particle case:

$$
\begin{aligned}
\underline{V}\left(\boldsymbol{b}_{1}, \boldsymbol{b}_{2}, \boldsymbol{b}_{3}\right)= & \underline{a_{21}}\left(\boldsymbol{b}_{2}-\boldsymbol{b}_{1}\right)^{2}+\underline{a}_{32}\left(\boldsymbol{b}_{3}-\boldsymbol{b}_{2}\right)^{2} \\
& +\underline{a}_{13}\left(\boldsymbol{b}_{1}-\boldsymbol{b}_{3}\right)^{2} .
\end{aligned}
$$

In the special case $\boldsymbol{b}_{1}=\boldsymbol{b}_{2}$, this gives

$$
\underline{V}\left(\boldsymbol{b}_{1}, \boldsymbol{b}_{1}, \boldsymbol{b}_{3}\right)=\left(\underline{a}_{32}+\underline{a}_{13}\right)\left(\boldsymbol{b}_{3}-\boldsymbol{b}_{1}\right)^{2} .
$$

However, in this case we have color charge $\mathbb{T}_{3}$ at $\boldsymbol{b}_{3}$ and, since $\mathbb{T}_{1}+\mathbb{T}_{2}+\mathbb{T}_{3}=0$, total color charge $\mathbb{T}_{1}+\mathbb{T}_{2}=-\mathbb{T}_{3}$ at $\boldsymbol{b}_{1}=\boldsymbol{b}_{2}$. This is then effectively a two-body problem, in the color representation of particle 3 , with separation $\Delta \boldsymbol{b}=\boldsymbol{b}_{3}-\boldsymbol{b}_{1}$. That means that

$$
\underline{V}\left(\boldsymbol{b}_{1}, \boldsymbol{b}_{1}, \boldsymbol{b}_{3}\right)=-\frac{i}{4} \hat{q}_{3}\left(\boldsymbol{b}_{3}-\boldsymbol{b}_{1}\right)^{2}
$$

in quadratic approximation, as in (2.12). Combined with (3.5), this gives a constraint $\underline{a}_{32}+\underline{a}_{13}=-\frac{i}{4} \hat{q}_{3}$ on the values of the coefficients $\underline{a}_{i j}$. Permuting the particle labels in this argument then provides three constraints on the three unknown coefficients $\underline{a}_{i j}$, which then uniquely determine the three-body potential to be (1.4) in harmonic approximation. For what follows, I will find it more useful to write that three-body potential in the form (1.5) that I will use for the $N$-body potential:

$$
\begin{aligned}
\underline{V}\left(\boldsymbol{b}_{1}, \boldsymbol{b}_{2}, \boldsymbol{b}_{3}\right)= & -\frac{i}{8}\left[\left(\hat{q}_{2}+\hat{q}_{1}-\underline{\hat{q}}_{21}\right)\left(\boldsymbol{b}_{2}-\boldsymbol{b}_{1}\right)^{2}\right. \\
& +\left(\hat{q}_{3}+\hat{q}_{2}-\underline{\hat{q}}_{32}\right)\left(\boldsymbol{b}_{3}-\boldsymbol{b}_{2}\right)^{2} \\
& \left.+\left(\hat{q}_{1}+\hat{q}_{3}-\underline{\hat{q}}_{13}\right)\left(\boldsymbol{b}_{1}-\boldsymbol{b}_{3}\right)^{2}\right] .
\end{aligned}
$$

As previously noted, in the three-body case, $\underline{\hat{q}}_{21}$ is the same as $\hat{q}_{3}$, and so forth.

\section{B. The $N$-particle case}

Now return to the generic form (3.3) for a harmonic oscillator approximation in the $N$-body case. Consider now the special case where we put all the particles but the first two at the same position $\boldsymbol{b}_{3}=\boldsymbol{b}_{4}=\cdots=\boldsymbol{b}_{N}$, so that (3.3) gives

$$
\begin{aligned}
V\left(\boldsymbol{b}_{1}, \boldsymbol{b}_{2}, \boldsymbol{b}_{3}, \boldsymbol{b}_{3}, \ldots, \boldsymbol{b}_{3}\right) \\
=\underline{a}_{21}\left(\boldsymbol{b}_{2}-\boldsymbol{b}_{1}\right)^{2}+\left(\sum_{j \geq 3} \underline{a}_{j 1}\right)\left(\boldsymbol{b}_{3}-\boldsymbol{b}_{1}\right)^{2} \\
\quad+\left(\sum_{j \geq 3} \underline{a}_{j 2}\right)\left(\boldsymbol{b}_{3}-\boldsymbol{b}_{2}\right)^{2} .
\end{aligned}
$$

On the other hand, $\boldsymbol{b}_{3}=\boldsymbol{b}_{4}=\cdots=\boldsymbol{b}_{N}$ is effectively a three-body problem where one particle has color generator $\underline{\mathbb{I}}_{3}+\cdots+\mathbb{\underline { I }}_{N}$. So (3.8) must be the same as the three-body potential (3.7) with the replacement $\underline{\mathbb{I}}_{3} \rightarrow \underline{\mathbb{I}}_{3}+\cdots+\underline{\mathbb{I}}_{N}$ 
in the latter. Focusing on the $\left(\boldsymbol{b}_{2}-\boldsymbol{b}_{1}\right)^{2}$ term for simplicity, that identification requires

$$
\underline{a}_{21}=-\frac{i}{8}\left(\hat{q}_{2}+\hat{q}_{1}-\underline{\hat{q}}_{21}\right) \text {. }
$$

There was nothing special about which of the $N$ particles we labeled as 1 and 2 in this argument, so generally

$$
\underline{a}_{i j}=-\frac{i}{8}\left(\hat{q}_{i}+\hat{q}_{j}-\underline{\hat{q}}_{i j}\right) .
$$

Substitution into (3.3) then gives the final result (1.5) of this paper for the $N$-body potential in harmonic approximation.

\section{CAVEATS AND CLARIFICATIONS}

\section{A. Caveats for $\hat{q}$ approximation}

\section{Logarithmic dependence}

The two-body potential, which I have called $V(\Delta b)$, is not precisely quadratic (1.2) in the small- $\Delta b$ limit. Instead, the coefficient $\hat{q}$ effectively depends logarithmically on $\Delta b$. One type of possible logarithmic dependence can be seen in leading-order calculations of $\hat{q}$, where $d \Gamma_{\mathrm{el}} / d^{2} q_{\perp} \propto \alpha^{2} / q_{\perp}^{4}$ for large $q_{\perp}$ : if one does not account for any running of the coupling constant $\alpha_{\mathrm{s}}$, the leading-order result for $\hat{q}(\Delta b)$ blows up logarithmically as $\Delta b \rightarrow 0$, so that $\hat{q}(0)$ as defined by (2.11) is ultraviolet (UV) divergent. If one instead uses the running coupling $\alpha_{\mathrm{s}}\left(q_{\perp}\right)$ when calculating (2.11), the log dependence of $\hat{q}(\Delta b)$ cuts off when $\alpha_{\mathrm{s}}(1 / \Delta b) \ll \alpha_{\mathrm{s}}\left(m_{\mathrm{D}}\right)$ (where $m_{\mathrm{D}}$ is a plasma scale, representing the Debye mass), and the leading-order result for $\hat{q}(0)$ is finite. ${ }^{6}$ But there remains other log dependence that cannot be seen at leading order [20], which I will later review in Sec. IV B 2.

The arguments in this paper (as most any application of the $\hat{q}$ approximation) rely on logarithmic dependence of $\hat{q}$ being mild enough that one can simply approximate the coefficient $\hat{q}$ by some fixed effective value relevant to the scale of a particular application.

\section{Applies to typical events}

Another issue with the $\hat{q}$ approximation is that $\hat{q}$ only determines the transverse momentum transfer for typical multiple scattering events. Because of large- $q_{\perp}$ tails to the probability distribution for momentum transfer in Coulomb scattering, there are also rarer events with scattering by larger-than-typical angles. Depending on the situation and what average quantity one is interested in calculating, atypical events can sometime dominate averages. ${ }^{7}$

\footnotetext{
${ }^{6}$ See, for example, Sec. VI. B of Ref. [18], which combines earlier observations of Refs. [8,19].

${ }^{7}$ See BDMPS, Sec. 3.1 of Ref. [8], and Zakharov [21]. Some further discussion is given in Ref. [22].
}

\section{The limit of lightlike Wilson lines}

Consider Wilson lines corresponding to particles with velocity $v$. The lightlike Wilson lines used in Wilson loops such as Fig. 1(a) to define $\hat{q}$ [or more generally $\hat{q}(\Delta b)$ ] correspond to the limiting case $v=1$. There have been some confusing subtleties in the literature on how to approach this limit-discussion which has been in the context of calculations of $\hat{q}$ in QCD-like theories with gravity duals and which is also related to issues of regularizing UV divergences associated with Wilson lines. Here, I want to make a few simple observations about the $v=1$ limit in the general context of gauge theories, and then I will draw some parallel to the issues in gauge-gravity duality calculations at the end.

Physically, the test particles represented by the long sides of the Wilson loop should have $v<1$, and so the lightlike limit represents approaching $v=1$ from below. A natural impulse is to hope that if the limit makes sense, then one should also be able to approach $v=1$ from above-that is, using Wilson loops whose long sides are (slightly) ${ }^{8}$ spacelike rather than (slightly) timelike. If so, then there are simple, direct arguments that the Wilson loops, and the potentials $V(b)$ defined by them, have the following very nice and relevant properties.

First, time-ordering prescriptions do not matter for gauge fields sourced by spacelike Wilson loops. In (all-orders) perturbative language, for example, consider a correlator

$$
\left\langle A_{\mu_{1}}^{a_{1}}\left(x_{1}\right) \cdots A_{\mu_{n}}^{a_{n}}\left(x_{n}\right)\right\rangle
$$

of gauge fields located at $n$ different points $x_{i}$ on the spatial Wilson loop. Because the $x_{i}$ are then all spacelike separated from each other, any operators at different $x_{i}$ must commute because of causality. So the ordering of the fields in the correlator (4.1) is irrelevant. That means we will get the same answer if we use time-ordered correlators, anti-time-ordered correlators, Wightman (unordered) correlators, SchwingerKeldysh correlators, or whatever. ${ }^{9}$

This is a significant property which has been implicitly assumed in applications of leading-order BDMPS-Z splitting rates to strongly coupled quark-gluon plasmas. If time ordering matters, then there is no general reason to think that correlations between medium interactions of the two blue lines (representing a pair of particles in the amplitude) in Fig. 2 is the same as the correlations between medium interactions of a blue and a red line (representing one particle in the amplitude and one in the conjugate amplitude).

\footnotetext{
${ }^{8}$ Here "slightly spacelike" means as considered in the plasma rest frame.

${ }^{9}$ Non-Abelian Wilson loops still have very important color ordering, represented by path ordering in color space of the exponential $P \exp \left(i g \oint_{C} A^{\mu} d x_{\mu}\right)$ defining the Wilson loop. This only affects how the color indices $a_{i}$ in (4.1) will be contracted.
} 
The derivation given in Sec. III A for the $\hat{q}$ approximation to the three-body potential (1.4) would then be invalid. That would in turn cast doubt on the applicability of the standard BDMPS-Z formula for $g \rightarrow g g$ splitting in $\hat{q}$ approximation in the case of strongly coupled plasmas (outside of the soft bremsstrahlung approximation, at least).

I should note that previous authors ${ }^{10}$ have made a point that $\hat{q}$ should be defined with Schwinger-Keldysh ordering, which is a natural choice when thinking of the meaning of $\hat{q}$ in terms of the rate for $\boldsymbol{p}_{\perp}$ broadening. But if this ordering distinction is actually important in the lightlike limit, we must then face the just-discussed difficulty when using $\hat{q}$ for BDMPS-Z splitting rates.

A related nice property, also easy to derive if one may approach lightlike Wilson loops $v=1$ from the limit of spacelike loops $v>1$, is that expectations of Wilson loops are real-valued, and so the potential $V(b)$ is pure imaginary as I assumed earlier. Correspondingly $\hat{q}$ defined by (1.2) is then real valued. One way to see this is that, if timeordering prescriptions do not matter for spacelike loops, then taking the complex conjugation of the Wilson loop $\operatorname{tr}\left[P \exp \left(i \oint_{C} A^{\mu} d x_{\mu}\right)\right]$ is equivalent to flipping the direction of integration around the loop, which is equivalent to rotating the loop by 180 degrees in the transverse plane. By rotational invariance, the result for the Wilson loop must therefore equal its complex conjugate and so is real.

In discussing details of their gauge-gravity duality calculation, Liu, Rajagopal, and Wiedemann [2] do not consider $v>1$. They characterize their calculation as approaching lightlike Wilson loops from the (physically motivated) $v<1$ side but note that they have to take the $v=1$ limit before they remove their UV regulator, which corresponds to a tiny displacement of their string end points into the fifth dimension. ${ }^{11}$ However, with their UV regulator in place, in the gravity description the end points of their strings are forced to move faster than the local speed of light (as noted in Ref. [25]), which is a reflection of the fact that the string world sheets they find are purely spacelike in the order of limits that they take. Their result is the same as they would get if they took their limit from the $v>1$ side.

If the limits of approaching $v=1$ from above and below are not the same for all applications of Wilson loop potentials to splitting rates, and furthermore if gauge field ordering prescriptions do matter in the lightlike limit, then one will have to figure out how to appropriately adjust both BDMPS-Z splitting rates and the more generalized discussion of this paper.

\footnotetext{
${ }^{10}$ See section VI of ref. [23]. (For a discussion of gauge invariance in the case of that ordering prescription, see also ref. [24].)

${ }^{11}$ Specifically, see the discussions of orders of limits in section III of ref. [2] and especially the conclusion of their section III. 3 .
}

\section{B. Time and distance scales for color dynamics}

Earlier, I postponed discussing what it means to talk about the joint color representation $\underline{\mathbb{T}}_{i}+\underline{\mathbb{T}}_{j}$ of two spatially separated particles $i$ and $j$.

\section{An oversimplified argument}

For this purpose, it is important to realize that the background fields of the plasma will have characteristic wavelengths and correlation lengths set by plasma scales (e.g., $1 / T$ or $1 / g T$ or $1 / g^{2} T$, etc., where $T$ represents the temperature). But the $\hat{q}$ approximation relevant to high-energy particles $(E \gg T)$ corresponds to $\Delta b$ small compared to plasma scales. That means that the different high-energy particles represented by the lightlike Wilson lines in the two-body or $N$-body potentials are so close to each other that, to first approximation, they will experience the same background gauge field $\left[A_{\text {plasma }}\left(\boldsymbol{b}_{i}, z=t\right) \simeq A_{\text {plasma }}\left(\boldsymbol{b}_{j}, z=t\right)\right] .{ }^{12}$ If two slightly separated Wilson lines $i$ and $j$ are experiencing identical gauge fields as they move through light-cone time $x^{+}$, then their color charges $\underline{\mathbb{T}}_{i}$ and $\underline{\mathbb{I}}_{j}$ will rotate the same way, and so the sum $\underline{\mathbb{T}}_{i}+\underline{\mathbb{T}}_{j}$ will only experience an overall color rotation. In this approximation, if one starts in a given color subspace corresponding to some irreducible representation of $\underline{\mathbb{T}}_{i}+\underline{\mathbb{T}}_{j}$, then one remains in that irreducible representation.

However, no matter how close the $\boldsymbol{b}_{i}$ are, there will be small differences in the background color fields experienced by the different particles, and these will slowly accumulate over time. ${ }^{13}$ The individual colors will decohere over a time known as the color decoherence time $t_{\text {decohere }}$, which is parametrically of size

$$
t_{\text {decohere }} \sim \frac{1}{\hat{q}(\Delta b)^{2}} .
$$

This is just the timescale $L$ for which the values $e^{-i V L}$ of the Wilson loops discussed in this paper [e.g., (1.1) with (1.2)] first become significantly different from one. It is also the same timescale as formation times in applications to inmedium splitting rates. The important point is that, in the limit of small $\Delta b$, the color decoherence time (4.2) is parametrically large compared to all plasma scales, which means that the irreducible color representations of $\underline{\mathbb{I}}_{i}+\underline{\mathbb{T}}_{j}$ only mix slowly compared to the correlation length $\xi$ of the medium.

\footnotetext{
${ }^{12}$ Technically, pursuing the argument in the language of gauge fields $A$ requires insisting that the choice of gauge respects the separation of physics scales, so that plasma gauge fields are smooth on small scales (i.e., $\ll 1 / T$ ). I assume that and proceed.

${ }^{13}$ For a discussion of the language of color decoherence in the context of in-medium radiative processes, see, for example, Ref. [26]. The parametric estimates [such as their (5.2)] are different because they consider the case of lines at some angle $\theta_{q \bar{q}}$ to each other, instead of the parallel lines with fixed separation $\boldsymbol{b}_{i j}$ relevant to defining the potential $V$ here.
} 


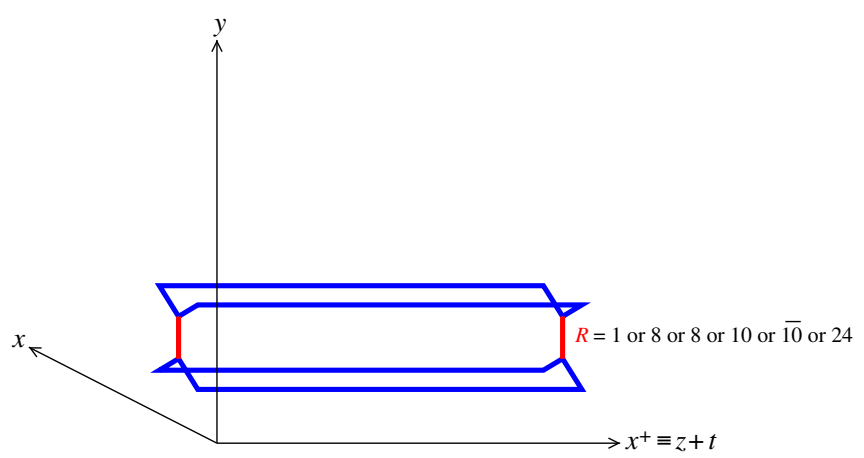

FIG. 7. An example of different ways to contract color for initial and final states in the case of an $\mathrm{N}$-body potential with $N>3$. The particular example above is for $N=4$ lightlike adjoint Wilson lines. Here, blue denotes adjoint representation. The two red line segments, however, may each be independently chosen to be in any irreducible representation $8 \otimes 8=1 \oplus 8 \oplus$ $8 \oplus 10 \oplus \overline{10} \oplus 27$. Because of the hierarchy of scales discussed in the text, the details of the lengths of the red lines, or the transverse positions of their end points, are not relevant to defining the potential $\underline{V}$ in the limit of small transverse separations because of the hierarchy of scales discussed in the text.

It is this hierarchy of timescales that makes it possible to consider color dynamics in the context of a potential approximation $V$. To define a potential, one needs Wilson loops that are long compared to the correlation length of the medium $(L \gg \xi)$. But since the color dynamics scale (4.2) is also long compared to the correlation length, it is then possible to treat that color dynamics in terms of a potential. One can imagine picking the initial and final color states by an appropriate contraction of the initial and final Wilson lines, as depicted, for example, in Fig. 7. Repeating this for all possible choices in some basis of possible color combinations would allow one to interpret the Wilson loops of Fig. 7 as giving a matrix result in color representation space that can then be written in exponential form $e^{-i \underline{V} L}$ to extract a corresponding matrix result for the potential $\underline{V}$ in that basis. We do not need to do any of this in practice, however, to obtain the result (1.5) of this paper. The point is just to understand why, for small transverse separations, it can be sensible to talk about the color structure of $\underline{V}$ in the first place.

\section{Splitting contributions}

The above discussion cheated somewhat, making an implicit assumption that ignored an additional complication: I assumed that the only way that the lightlike Wilson lines interact with the plasma is by directly experiencing fields already present in the plasma. However, there are also important contributions [20] where, instead, a lightlike Wilson line emits a high-energy $(\omega \gg T)$ nearly collinear gluon, which propagates a long time $\Delta t \gg \xi$ (scattering from the medium the whole time) before reattaching to a Wilson line. An example is shown in Fig. 8. As I will

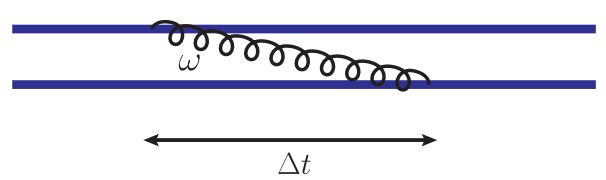

FIG. 8. A contribution where two lightlike Wilson lines are connected by a high-energy $(\omega \gg T)$, nearly collinear gluon line. Though not drawn explicitly above, the high-energy Wilson lines and gluon are all interacting repeatedly with the background fields of the plasma.

review, these processes either (i) can be absorbed into the potential $V$ or else (ii) are suppressed by the running coupling $\alpha_{\mathrm{s}}(\mu)$ evaluated at momentum scale $\mu \sim 1 / \Delta b$. Even if the plasma is strongly coupled, $\alpha_{\mathrm{s}}(1 / \Delta b)$ will be small for small enough $\Delta b$ (which, formally at least, is the relevant limit for applications to LPM splitting rates at large enough energy).

It was shown by Liou, Mueller, and $\mathrm{Wu}$ [20] that processes such as Fig. 8 generate corrections $\delta \hat{q}$ to $\hat{q}$ of size $\alpha_{\mathrm{s}} \hat{q}$ times a large double logarithm. In the context of the two-body potential, this corresponds to corrections $\delta V$ to the naive harmonic potential (1.2) of $\operatorname{size}^{14}$

$$
\begin{aligned}
\delta V(\Delta b) & \sim \alpha_{\mathrm{s}} \hat{q}(\Delta b)^{2} \ln ^{2}\left(\frac{t_{\text {decohere }}}{\tau_{0}}\right) \\
& \sim \alpha_{\mathrm{s}} \hat{q}(\Delta b)^{2} \ln ^{2}\left(\frac{1}{\hat{q} \tau_{0}(\Delta b)^{2}}\right),
\end{aligned}
$$

where $\tau_{0}$ is a characteristic scale of the medium that, for weakly coupled quark-gluon plasmas, corresponds to the mean free path for elastic scattering. Various authors [11-13] have shown that the double-log correction to $\hat{q}$ is universal in the sense that, if one computes similar double-log corrections to QCD LPM splitting rates (related to three-body potentials as in Fig. 3), then those corrections are also completely accounted for by the correction to $\hat{q}$ originally found by Liou et al. [20]. They were also able to sum leading logarithms at all orders in $\alpha_{\mathrm{s}}$, but the points I need to make here can already be discussed in the simpler context of (4.3).

Now consider what effect these double-log contributions have on my argument about the slow rate of mixing of different color representations for $\underline{\mathbb{I}}_{1}+\underline{\mathbb{I}}_{2}$ for $N$-body potentials. The logarithms in (4.3) arise from time separations $\Delta t$ in Fig. 8 over the parametrically large range

$$
\tau_{0} \ll \Delta t \ll t_{\text {decohere }}
$$

in concert with gluon energies $\omega$ in the range

\footnotetext{
${ }^{14}$ The double log shown in (4.3) assumes that the length $L$ of the Wilson loop is taken to infinity for fixed $\Delta b$. A more general parametric estimate would be to replace the argument $1 / \hat{q} \tau_{0}(\Delta b)^{2}$ of the $\log$ in (4.3) by $\min \left(t_{\text {decohere }}, L\right) / \tau_{0}$. For $\Delta b \rightarrow 0$ with fixed $L$, one then recovers the $\ln ^{2}\left(L / \tau_{0}\right)$ in Eq. (45) of Ref. [20], where my $\tau_{0}$ is their $l_{0}$.
} 


$$
\hat{q}(\Delta t)^{2} \ll \omega \ll(\Delta t) /(\Delta b)^{2}
$$

[which together restrict $\omega$ to $T \ll \omega \ll(\Delta t) /(\Delta b)^{2}$, where I have interpreted the plasma scale $\hat{q} \tau_{0}^{2}$ as order $\left.T\right]{ }^{15}$ Because $\Delta t \ll t_{\text {decohere, }}$, that means that the gluon exchange shown in Fig. 8 is local compared to the time

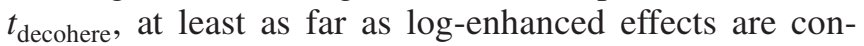
cerned. Earlier, when only considering the direct interactions of Wilson lines with plasma fields, I asserted that making sense of the color representation of $\underline{\mathbb{I}}_{1}+\underline{\mathbb{T}}_{2}$ required the hierarchy of scales $t_{\text {decohere }} \gg \xi$ so that the rate of color change was slow compared to the timescales of the physics generating the potential $V$. But the same argument can still be made for double-log contributions such as Fig. 8 because we have $t_{\text {decohere }} \gg \Delta t$.

One might next be concerned about effects from Fig. 8 that are not log enhanced, specifically the contribution from the upper end point $\Delta t \sim t_{\text {decohere }}$ of the range (4.4). In this case, there is no separation of scale. The corresponding contribution to the potential is of order (4.3) without the double $\log$ factor,

$$
\delta V(\Delta b) \sim \alpha_{\mathrm{s}} \hat{q}(\Delta b)^{2},
$$

which is suppressed by an uncompensated factor of $\alpha_{\mathrm{s}}$ compared to the potential (1.2). That would not really be a "suppression" if the scale for that $\alpha_{\mathrm{s}}$ were a plasma scale, e.g., $\alpha_{\mathrm{s}}\left(m_{\mathrm{D}}\right)$, since the whole point is to be able to discuss strongly coupled plasmas. However, the explicit $\alpha_{\mathrm{s}}$ in (4.5) arises from the two factors of $g$ associated with where the high-energy gluon connects to the Wilson lines in Fig. 8. The relevant distance scale for this coupling is the typical relative separation $\Delta B_{\perp}$ of the gluon from the Wilson lines in Fig. 8, which can be estimated from free gluon diffusion ${ }^{16}$ as $\Delta B_{\perp} \sim \sqrt{(\Delta t) / \omega}$. In the limiting case $\Delta t \sim t_{\text {decohere }}$ now

\footnotetext{
${ }^{15} \mathrm{I}$ have assumed that $L$ is large compared to all other distance scales in (4.4). For readers who may find these ranges more familiar or understandable in terms of other variables, here is a translation. (Translation 1) I have assumed $\Delta b$ nonzero and $L \gg$ $t_{\text {decohere }}$ in my analysis, but many analyses instead study the case of $\Delta b \rightarrow 0$ with $L$ fixed, for which $t_{\text {decohere }} \gg L$. One can get the parametric ranges in that case from the crossover case $t_{\text {decohere }} \sim L$. From (4.2), the crossover case corresponds to $\Delta b \sim(\hat{q} L)^{-1 / 2}$, and so (4.4) becomes $\tau_{0} \ll \Delta t \ll L$ and $\hat{q}(\Delta t)^{2} \ll \omega \ll \hat{q} L \Delta t$, which together restrict $T \ll \omega \ll \omega_{\mathrm{c}} \equiv \hat{q} L^{2}$. (Translation 2) If interested instead in the application to LPM bremsstrahlung of a gluon with energy $\Omega$ in an arbitrarily thick plasma $(L \rightarrow \infty)$, one may again use the first translation but replace $L$ by the formation time for the underlying bremsstrahlung, which is of order $t_{\text {form }}(\Omega) \sim \sqrt{\Omega / \hat{q}}$. That is equivalent to setting $t_{\text {decohere }} \sim t_{\text {form }}(\Omega)$. The typical size of $\Delta b$ for the underlying bremsstrahlung is then of order $\Delta b \sim\left(\hat{q} t_{\text {form }}\right)^{-1 / 2} \sim(\hat{q} \Omega)^{-1 / 4}$. The ranges (4.4) [now representing double-log effects from additional bremsstrahlung (real or virtual) of a second gluon $(\omega)$ from the original gluon $(\Omega)$ ] are $\tau_{0} \ll \Delta t \ll$ $t_{\text {form }}(\Omega)$ and $\hat{q}(\Delta t)^{2} \ll \omega \ll \hat{q} t_{\text {form }}(\Omega) \Delta t$, which together restrict $T \ll \omega \ll \Omega$.

${ }^{16}$ See the discussion of Eq. (13) of Liou et al. [20].
}

being discussed, this is $\Delta B_{\perp} \sim \Delta b$. So, in the limit of small $\Delta b$, the troublesome $\Delta t \sim t_{\text {decohere }}$ corrections to the potential are proportional to $\alpha_{\mathrm{s}}(1 / \Delta b)$ and so are indeed suppressed for small enough $\Delta b$. A recent discussion of this scale for $\alpha$ in the application to overlapping formation times beyond double-log approximation (a discussion related to the fourbody potential) can be found in Ref. [27].

\section{CONCLUSION}

The result of this paper (1.5) provides the equivalent of the $\hat{q}$ approximation but for $N$-body potentials defined with parallel, lightlike Wilson lines. Such potentials will be needed, for example, to study overlapping formation times during in-medium shower development without taking the large- $N_{\mathrm{c}}$ limit. The core argument, given in Sec. III, is short and simple; the rest of this paper just frames it with the necessary background. In particular, the $N$-body potential has a nontrivial color structure for $N>3$. A discussion of how this structure could be implemented in calculations of overlapping formation times for three-color (as opposed to large- $N_{\mathrm{c}}$ ) QCD requires additional machinery and is left for later work [28].

\section{ACKNOWLEDGMENTS}

I am greatly indebted to Han-Chih Chang, Simon CaronHuot, Guy D. Moore, Hong Liu, Gunnar Bali, and Yacine Mehtar-Tani for valuable discussions. This work was supported, in part, by the U.S. Department of Energy under Grant No. DE-SC0007984.

\section{APPENDIX: TWO-POINT GAUGE CORRELATORS AND $\Gamma_{\mathrm{el}}(b)$}

In this appendix, I review how two-point correlators between the two lightlike Wilson lines in Fig. 5 are given by the $\Gamma_{\mathrm{el}}(\boldsymbol{b})$ of (2.4). Many of the original discussions of the physics of $\hat{q}$ and QCD LPM splitting rates in the literature assume particular models for interactions with the medium such as Debye-screened Coulomb scatterings from static rather than dynamic scattering centers, ignoring the dependence of scattering cross sections on the momentum (and so momentum distribution) of plasma particles, and/or assuming that individual momentum exchanges $q$ are soft compared to the plasma temperature $T$. But I will instead keep the discussion here general.

Let $x$ be a point on one lightlike Wilson line and $y$ on the other. First note that these two points are then spacelike separated, which means (importantly) that we will not need to worry about time ordering of the gauge fields $A(x)$ and $A(y)$ in what follows. Now use translation invariance to fix $y$ at the origin and integrate $x$ along its Wilson line $\left(x^{0}, x^{1}, x^{2}, x^{3}\right)=(t, b, t)$ to give the correlator

$$
\mathcal{C} \equiv g^{2} \int d t\langle v \cdot A(t, \boldsymbol{b}, t) v \cdot A(0)\rangle
$$


where

$$
v^{\mu} \equiv(1,0,0,1)
$$

Rewriting $\langle v \cdot A(x) v \cdot A(0)\rangle$ in terms of its Fourier transform and then performing the $d t$ integral above gives

$$
\mathcal{C}=g^{2} \int_{q} e^{i \boldsymbol{q}_{\perp} \cdot \boldsymbol{b}}\left\langle v \cdot \tilde{A}(q)^{*} v \cdot \tilde{A}(q)\right\rangle 2 \pi \delta(v \cdot q)
$$

where the integral is over four-momentum $q$. Let $|\mathrm{i}\rangle$ be any possible state of the medium. The above is then the appropriate medium-state average of ${ }^{17}$

$$
\mathcal{C}=g^{2} \int_{q} e^{i \boldsymbol{q}_{\perp} \cdot \boldsymbol{b}}\left\langle\mathrm{i}\left|v \cdot \tilde{A}(q)^{*} v \cdot \tilde{A}(q)\right| \mathrm{i}\right\rangle 2 \pi \delta(v \cdot q) .
$$

Inserting a complete set of intermediate states $|\mathrm{f}\rangle$ in the middle,

$$
\mathcal{C}=\int_{q} e^{i q_{\perp} \cdot b} \sum_{\mathrm{f}}|\langle\mathrm{f}|g v \cdot \tilde{A}(q)| \mathrm{i}\rangle|^{2} 2 \pi \delta(v \cdot q) .
$$

\footnotetext{
${ }^{17}$ For example, for thermal equilibrium, take $|\mathrm{i}\rangle$ to be exact quantum field theory energy eigenstates and average (A4) with relative weight $e^{-\beta E_{\mathrm{i}}}$. One may do something similar with any density matrix describing the medium by working in the basis where the density matrix is diagonal.
}

Now consider instead calculating the elastic scattering rate of a particle with very high energy $E$ via exchanging a gluon with the medium. In the limit that $E$ is much higher than the exchanged momentum $q$, the particle-gluon vertex is $i g v \cdot A$ times a relativistic normalization factor of $2 E$. The rate is then

$\Gamma_{\mathrm{el}}=\sum_{\mathrm{f}} \int_{q} \frac{1}{2 E}|\langle\mathrm{f}|g v \cdot \tilde{A}(q) 2 E| \mathrm{i}\rangle|^{2} 2 \pi \delta\left((P+q)^{2}\right)$,

where $P \equiv(E, 0,0, E)$ is the high-energy particle's fourmomentum, $1 / 2 E$ is the usual initial-state normalization factor, and $\delta\left((P+q)^{2}\right)$ puts the final state of the highenergy particle on shell. Remember that the states $|\mathrm{i}\rangle$ and $|\mathrm{f}\rangle$ above refer to states of the medium and do not include the states of the high-energy particle, which here have been treated explicitly. Making use of the high-energy limit for the $P$ inside the $\delta$ function,

$$
\Gamma_{\mathrm{el}}=\sum_{\mathrm{f}} \int_{q}|\langle\mathrm{f}|g v \cdot \tilde{A}(q)| \mathrm{i}\rangle|^{2} 2 \pi \delta(v \cdot q) .
$$

Dropping the $\boldsymbol{q}_{\perp}$ integration above,

$$
\frac{d \Gamma_{\mathrm{el}}}{d^{2} q_{\perp}}=\sum_{\mathrm{f}} \int_{q^{0}, q^{2}}|\langle\mathrm{f}|g v \cdot \tilde{A}(q)| \mathrm{i}\rangle|^{2} 2 \pi \delta(v \cdot q) .
$$

Plugging this into the definition (2.4) of $\Gamma_{\mathrm{el}}(\boldsymbol{b})$ then shows that the correlator $\mathcal{C}$ (A5) between two Wilson lines is the same as $\Gamma_{\mathrm{el}}(\boldsymbol{b})$.
[1] H. Liu, K. Rajagopal, and U. A. Wiedemann, Calculating the Jet Quenching Parameter from AdS/CFT, Phys. Rev. Lett. 97, 182301 (2006).

[2] H. Liu, K. Rajagopal, and U. A. Wiedemann, Wilson loops in heavy ion collisions and their calculation in AdS/CFT, J. High Energy Phys. 03 (2007) 066.

[3] S. Caron-Huot, $\mathrm{O}(\mathrm{g})$ plasma effects in jet quenching, Phys. Rev. D 79, 065039 (2009).

[4] C. Anzai, Y. Kiyo, and Y. Sumino, Violation of Casimir scaling for static QCD potential at three-loop order, Nucl. Phys. B838, 28 (2010); Erratum, Nucl. Phys.B890, 569(E) (2015).

[5] R. N. Lee, A. V. Smirnov, V. A. Smirnov, and M. Steinhauser, Analytic three-loop static potential, Phys. Rev. D 94, 054029 (2016).

[6] A. Grozin, J. Henn, and M. Stahlhofen, On the Casimir scaling violation in the cusp anomalous dimension at small angle, J. High Energy Phys. 10 (2017) 052.

[7] R. Baier, Y. L. Dokshitzer, A. H. Mueller, S. Peigne, and D. Schiff, The Landau-Pomeranchuk-Migdal effect in QED,
Nucl. Phys. B478, 577 (1996); Radiative energy loss of high-energy quarks and gluons in a finite volume quarkgluon plasma, Nucl. Phys. B483, 291 (1997).

[8] R. Baier, Y. L. Dokshitzer, A. H. Mueller, S. Peigne, and D. Schiff, Radiative energy loss and $p_{\perp}$-broadening of high energy partons in nuclei, Nucl. Phys. B484, 265 (1997).

[9] B. G. Zakharov, Fully quantum treatment of the LandauPomeranchuk-Migdal effect in QED and QCD, JETP Lett. 63, 952 (1996); Radiative energy loss of high-energy quarks in finite size nuclear matter an quark-gluon plasma, Pis'ma Zh. Eksp. Teor. Fiz. 65, 585 (1997) [JETP Lett. 65, 615 (1997)].

[10] P. Arnold and S. Iqbal, The LPM effect in sequential bremsstrahlung, J. High Energy Phys. 04 (2015) 070; Erratum, J. High Energy Phys.09 (2016) 072(E).

[11] J. P. Blaizot and Y. Mehtar-Tani, Renormalization of the jetquenching parameter, Nucl. Phys. A929, 202 (2014).

[12] E. Iancu, The non-linear evolution of jet quenching, J. High Energy Phys. 10 (2014) 95. 
[13] $\mathrm{B}$. Wu, Radiative energy loss and radiative $p_{\perp}$-broadening of high-energy partons in QCD matter, J. High Energy Phys. 12 (2014) 081.

[14] P. Arnold, H. C. Chang, and S. Iqbal, The LPM effect in sequential bremsstrahlung 2: Factorization, J. High Energy Phys. 09 (2016) 078.

[15] P. Arnold, H. C. Chang, and S. Iqbal, The LPM effect in sequential bremsstrahlung: Dimensional regularization, J. High Energy Phys. 10 (2016) 100.

[16] P. Arnold, H. C. Chang, and S. Iqbal, The LPM effect in sequential bremsstrahlung: 4-gluon vertices, J. High Energy Phys. 10 (2016) 124.

[17] P. B. Arnold, Simple formula for high-energy gluon bremsstrahlung in a finite, expanding medium, Phys. Rev. D 79, 065025 (2009).

[18] P. B. Arnold and C. Dogan, QCD splitting/joining functions at finite temperature in the deep LPM regime, Phys. Rev. D 78, 065008 (2008).

[19] A. Peshier, QCD running coupling and collisional jet quenching, J. Phys. G 35, 044028 (2008).

[20] T. Liou, A. H. Mueller, and B. Wu, Radiative $p_{\perp}$-broadening of high-energy quarks and gluons in QCD matter, Nucl. Phys. A916, 102 (2013).
[21] B. G. Zakharov, On the energy loss of high-energy quarks in a finite size quark-gluon plasma, Pis'ma Zh. Eksp. Teor. Fiz. 73, 55 (2001) [JETP Lett. 73, 49 (2001)].

[22] P. B. Arnold, High-energy gluon bremsstrahlung in a finite medium: Harmonic oscillator versus single scattering approximation, Phys. Rev. D 80, 025004 (2009).

[23] F. D'Eramo, H. Liu, and K. Rajagopal, Transverse momentum broadening and the jet quenching parameter, redux, Phys. Rev. D 84, 065015 (2011).

[24] M. Benzke, N. Brambilla, M. A. Escobedo, and A. Vairo, Gauge invariant definition of the jet quenching parameter, J. High Energy Phys. 02 (2013) 129.

[25] H. Liu, K. Rajagopal, and Y. Shi, Robustness and infrared sensitivity of various observables in the application of AdS/CFT to heavy ion collisions, J. High Energy Phys. 08 (2008) 048.

[26] Y. Mehtar-Tani and K. Tywoniuk, Jet coherence in QCD media: The antenna radiation spectrum, J. High Energy Phys. 01 (2013) 031.

[27] P. Arnold, S. Iqbal, and T. Rase, Strong- vs. weak-coupling pictures of jet quenching: A dry run using QED, arXiv: 1810.06578 .

[28] P. Arnold and H. C. Chang (to be published). 